\title{
Dynamic Pricing of New Products in Competitive Markets: A Mean-Field Game Approach
}

\author{
Régis Chenavaz, Corina Paraschiv $\dagger$ Gabriel Turinici ${ }^{\ddagger}$
}

September 25, 2017

\begin{abstract}
Dynamic pricing of new products has been extensively studied in monopolistic and oligopolistic markets. But, the optimal control and differential game tools used to investigate the pricing behavior on markets with a finite number of firms are not wellsuited to model competitive markets with an infinity of firms. Using a mean-field games approach, this paper examines dynamic pricing policies in competitive markets, where no firm exerts market power. The theoretical setting is based on a diffusion model $\grave{a} l a$ Bass. We prove both the existence and the uniqueness of a mean-field game equilibrium, and we investigate mean tendencies and firms dispersion in the market. Numerical simulations show that the competitive market splits into two separate groups of firms depending on their production experience. The two groups differ in price and profit. Thus, high prices and profits do not have to signal anticompetitive practices, stimulating the debate on market regulation.
\end{abstract}

Keywords: Dynamic pricing, new products diffusion, mean-field games, competitive markets JEL Code: C61, C62, C73, D21, D41

\section{Introduction}

Dynamic pricing of new products has been widely examined in both monopolistic (Robinson and Lakhani, 1975; Kalish, 1983; Chen and Jain, 1992; Raman and Chatterjee, 1995) and oligopolistic settings (Dockner and Jørgensen, 1988; Dockner et al., 2000). In such settings, firms benefit from market power, and act as price makers. Markets with a finite number

\footnotetext{
*Kedge Business School, AMSE-GREQAM, CNRS \& EHESS

†Université Paris Descartes \& Institut Universitaire de France

‡Université Paris-Dauphine, PSL Research University, CNRS UMR 7534, CEREMADE, 75016 PARIS, FRANCE \& Institut Universitaire de France
} 
of firms (as in a dynamic monopoly and oligopoly) can be modeled with the classical tools of optimal control and differential games (see Kalish (1983) and Dockner and Jørgensen (1988)). These tools, however, are not designed to model competitive markets. Competitive markets are characterized by numerous firms that do not enjoy market power, and behave as price takers. They approach oligopoly markets (i.e., computers, mobile phones, printers) in which the number of firms is "large enough" (Weintraub et al., 2008, 2011; Adlakha and Johari, 2013a). Yet, differential games fail to provide tractable solutions in settings where the number of competing firms is "moderate" (Weintraub et al., 2008, 2011; Adlakha and Johari, 2013a). Due to technical complexity (Adlakha and Johari, 2013a) and the lack of appropriate tools, explicit analyses of competitive markets are missing (see the surveys by Chen and Chen (2015) and Den Boer (2015a)).

Mean field games (MFG) allow to investigate the behavior of markets with an infinity of players. MFG have been developed by Lasry and Lions (2006a,b) and Lasry and Lions (2007) in applied mathematics and independently by Huang et al. (2005, 2006) and Huang et al. (2007) in computer science. Describing MFG, Lasry and Lions (2007, p. 230) state "We introduce a general mathematical modelling approach to situations which involve a great number of 'agents.' Roughly speaking, we derive these models from a 'continuum limit' (in other words letting the number of agents go to infinity)." MFG allow modeling the interactions of numerous firms, while the MFG equilibrium represents a tractable solution approximating the oligopoly case (Weintraub et al., 2008, 2011; Adlakha et al., 2013; Adlakha and Johari, 2013a; Yang and Xia, 2013; Iyer et al., 2014).

Applying MFG, this paper analyzes the dynamic pricing policies of firms selling new products in competitive markets. The theoretical framework rests on a sales modeling $\grave{a}$ la Bass (1969), with diffusion and saturation effects on the demand side and learning effects on the supply side; each firm influences its sales with its pricing policy over time (Robinson and Lakhani, 1975; Kalish, 1983; Dockner and Jørgensen, 1988; Chen and Jain, 1992; Raman and Chatterjee, 1995; Krishnan et al., 1999; Dockner et al., 2000; Chatterjee, 2009). We model dynamic pricing competition with a continuum of firms where each is infinitesimal and no firm enjoys market power. The literature on the dynamic pricing of new products and on the application of MFG informs this research.

Studies on dynamic pricing originate from Bass (1969), who modeled a monopoly's sales of a new product accounting for diffusion and learning effects. The influence of pricing on sales is modeled with numerical methods by Robinson and Lakhani (1975), while analytical derivations of dynamic pricing rules appear in Kalish (1983) for the monopoly case, and in Dockner and Jørgensen (1988) for the oligopoly case. Dynamic pricing is further studied in Gallego and Van Ryzin (1997) for multi-products, in Gallego and Talebian (2012) for multi-version products, and in Gallego and $\mathrm{Hu}$ (2014) for substitutable and complementary products. Helmes et al. (2013) integrate dynamic advertising to address marketing-mix concerns. Danaher et al. (2001), followed by Jiang and Jain (2012), characterize the marketing-mix of successive generations of products. Gutierrez and He (2011) examine coordination between a retailer and a manufacturer for new product launchs, while Chutani and Sethi (2012) investigate promo- 
tional issues. Inventory considerations affecting dynamic pricing are also addressed (see the survey of Elmaghraby and Keskinocak (2003)). Jing (2011) investigates dynamic pricing with product diffusion and social learning, Chen and Farias (2013) consider imperfect forecast, Spann et al. (2014) complex markets, and Den Boer (2015b) investigates changing environments. However, while dynamic pricing has been widely studied in dynamic monopoly and oligopoly markets where firms enjoy market power (Chan and Sircar, 2015; Den Boer, 2015a), little is known about dynamic competitive markets, where a large number of firms set prices without benefiting from market power. The MFG tool appears well-suited for this purpose.

Early MFG applications mainly concern economics; examples include Lachapelle et al. (2010) and Guéant et al. (2011). Economic growth is addressed in relation to human capital (Lasry et al., 2008) and time allocation (Lucas and Moll, 2013). Production has been analyzed for an exhaustible resource (Bauso et al., 2012) and energy goods (Chan and Sircar, 2015). Financial markets have received attention, with Lachapelle et al. (2013) for price formation, and Carmona et al. (2013) for systematic risk. Gomes et al. (2014) study both consumer choice behavior and paradigm shifts in science. Adlakha et al. (2013) and Adlakha and Johari (2013a) examine industrial organization. Yang and Xia (2013) study revenue management, while Iyer et al. (2014) investigate auctions with learning. MFG have also been applied to population dynamics, covering crowd formation (Lachapelle and Wolfram, 2011) and vaccination decisions (Laguzet and Turinici, 2015; Salvarani and Turinici, 2017; Laguzet et al., 2016). Thus, MFG applications are burgeoning, attesting to a promising tool, particularly when applied to the dynamic pricing of new products.

This paper contributes to extant literature with dynamic pricing schemes for new products in competitive markets using MFG. The main results are as follows. We prove the existence and the uniqueness of a Nash-MFG equilibrium. We derive the equilibrium properties for pricing policies over time and across firms. MFG also allow to characterize analytically market heterogeneity. Numerical simulations show that market dynamics makes appear two groups of firms: the first with high prices, low sales, and low profits, the second with low prices, high sales, and high profits. Differences in prices and profits result from strong competition among numerous firm, which cannot extract rents from consumers. As such, greater (or lower) prices and profits donnot have to come from non-competitive practices. This implication calls for a greater carefulness from administrative authorities when they suspect non-competitive behaviors.

The rest of the article is organized as follows. Section 2 reviews elements of dynamic pricing of new products and MFG research, further explaining the main ingredients of the model. Section 3 describes the dynamic pricing model in a competitive market, which is resolved in Section 4. In Section 5, we examine the existence and uniqueness of the MFG equilibrium, the stability, and the monotonicity properties of optimal pricing policies, as well as the role of cumulative production in driving divergences in firm profits. Section 6 presents the numerical simulations and Section 7 concludes. 


\section{Related Contributions}

This section accounts for research in dynamic pricing of new products and MFG applications. We briefly highlight contributions in these fields that directly inform this research, and we end up by presenting the main model characteristics.

\subsection{Dynamic Pricing of New Products}

A major part of dynamic pricing research originates from the Bass (1969) new product diffusion model. The cumulative demand $x$ faced by a monopoly evolves at time $t$ as $d x / d t=(a+b x)(N-x)$, with $a, b, N>0$. The current demand $d x / d t$ increases with innovation $a$ and imitation $b$, and decreases with market saturation $N-x$ (with $N$ reflecting the total expected market for the product). In the Bass model, demand remains "passive," in the sense that price exerts no role.

Robinson and Lakhani (1975) enrich the analysis of new product diffusion by incorporating price sensitivity, allowing for a dynamic pricing examination. Demand depends on price $p$ as:

$$
\frac{d x}{d t}=(a+b x)(N-x) e^{-d p}
$$

with the price sensitivity of demand $d>0$. Firm's experience is captured through the reduction of the unit production cost $c$ with firm's cumulative (past) demand:

$$
c=c_{0}\left(x_{0} / x\right)^{\alpha},
$$

where $c_{0}$ reflects the firm's initial cost, $x_{0}$ its initial demand, and $\alpha \geq 0$. Kalish (1983) further generalizes the parametric formulation in (2.1) and (2.2) to a structural formulation, where demand writes $d x / d t=f(x, p)$ and the unit cost $c=c(x)$.

Our dynamic pricing model builds on Bass (1969), Robinson and Lakhani (1975), and Kalish (1983). We consider the diffusion, innovation, imitation, saturation, and experience effects. On this basis, we propose both structural and parametric analyses of dynamic pricing in competitive markets.

Furthermore, Dockner and Jørgensen (1988) generalize monopolistic dynamic pricing to an oligopoly market with a finite number $M$ of competing firms. The current demand of oligopoly $i(i=1, \ldots, M)$ depends on its price $p_{i}$, on its cumulative demand $x_{i}$, as well as the prices and cumulative demand of other firms:

$$
\frac{d x_{i}}{d t}=f^{i}\left(x_{1}, \ldots, x_{M}, p_{1}, \ldots, p_{M}\right)
$$

with the firm's unit production cost depending only on its own cumulative past demand: $c^{i}=c^{i}\left(x_{i}\right)$. The general formulation in equation (2.3) allows studying particular cases, such 
as multiplicatively separable demand $d x_{i} / d t=h^{i}\left(x_{i}\right) g^{i}\left(p_{1}, \ldots, p_{M}\right)$, and modelling a pricedifferential effect with $g^{i}\left(p_{1}, \ldots, p_{M}\right)=a_{i}-d_{i} p_{i}+k\left(p_{i}-\bar{p}\right)$, where $\bar{p}$ is the average market price and $k>0$.

Our dynamic pricing model generalizes Dockner and Jørgensen (1988), by transposing the oligopoly market with a finite number of firms into a competitive market with an infinite number of firms. To model competitive markets we use an MFG framework.

\subsection{Mean-Field Games}

The classic approach to analyze dynamic games considers a finite number of players (Fudenberg and Tirole, 1991). With a finite number of players, the current decision of each player depends on the current state of all players. Several authors (Adlakha and Johari, 2013b; Iyer et al., 2014; Adlakha et al., 2015) note the weakness of this approach when the number of players increases. First, increased complexity of the equilibrium may make it untractable. ${ }^{1}$ Indeed, equilibrium requires solving a coupled system of nonlinear partial differential equations, with a value function assigned to each player (Chan and Sircar, 2015). Second, postulating that each player takes into account the behavior of all other players is a strong requirement raising plausibility concerns (Adlakha et al., 2015); each player is thus assumed to track and integrate the state of all other players at any moment in time.

The MFG approach has been developed to deal with situations where the number of players is infinite (or tends to infinity) (Lasry and Lions, 2006a,b; Lasry and Lions, 2007; Huang et al., 2005, 2006, 2007). A mean-field denotes the distribution of a random variable, captured in the MFG model through different indexes, such as mean or variance. An MFG equilibrium is both simpler and more credible than an equilibrium with a finite number of players (Adlakha and Johari, 2013b; Iyer et al., 2014; Adlakha et al., 2015).

From a mathematical perspective, when the number of players tends to infinity, the analytic properties of the asymptotic regime simplify the analysis. Such simplification based on the law of large numbers drives a MFG equilibrium. MFG are characterized by a coupled system of (only) two partial differential equations (Guéant et al., 2011; Bensoussan et al., 2013; Carmona and Delarue, 2018). A backward Hamilton-Jacobi-Bellman equation captures the optimizing behavior of players; a forward Kolmogorov equation (also known as Fokker-Planck equation) accounts for the evolution of the mean field (Chan and Sircar, 2015). Further, MFG equilibrium possesses the property of approximating ${ }^{2}$ a Nash equilibrium when numerous players exert influence (see Adlakha and Johari (2013b), Iyer et al. (2014), Adlakha et al. (2015), and Chan and Sircar (2015) for MFG equilibria approximating Nash equilibria).

\footnotetext{
${ }^{1}$ Weintraub et al. (2008, p. 1377) affirm that "most industries contain more than 20 firms, but it would require more than 20 million gigabytes of computer memory to store the policy function for an industry with just 20 firms and 40 states".

${ }^{2}$ Technically, an MFG equilibrium approximates a Nash equilibrium with a finite but large number of players. The approximation process is the following. First, optimization of the game with a finite number of players, second the passage to the limit. Note that the steps are not commutative.
} 
From an economic perspective, the MFG approach simplifies the analysis. Indeed, the mean-field synthesizes all information about a variable distribution relevant for players making a decision. Players take a mean-field as given, and behave dynamically while fixing this mean field (Adlakha and Johari, 2013b). As players react "only" to the mean field, they disregard the situation and the actions of every other player (opposing a Nash approach, which entails much more sophisticated and less realistic players). MFG also enable players to form rational expectations. That is, players correctly anticipate the evolution of the mean-field (thanks to the backward equation). One of the strength of MFG is providing a computational simplification at the macroscopic level, while still recognizing the microstructure of the problem. The power of the MFG tool has led to continuously increasing applications (see Gomes and Saúde (2014) survey).

\subsection{Model Characteristics}

This paper applies MFG to model the dynamic pricing behavior of firms in competitive markets. Hereafter, we resume and briefly discuss the main characteristics of the model.

1. Market Structure. We consider a market with an infinite number of firms pricing an homogeneous product to an infinite number of consumers over time. In other words, we model a dynamic non-atomic game with a continuity of firms and consumers. Each firm sells a finite number of goods, making a finite profit. The market is competitive in the sense that no single firm influences market conditions and extracts rent from consumers. However, firms may price differently identical products due to different locations of consumers and the implied transportation costs (see also Yang and Xia (2013, p. 91) for similar assumptions). Nevertheless, the market conditions still depend on firms' prices in aggregation.

2. Firm Behavior. Firms share a simple representation of the world. They are not assumed to be overly sophisticated in the sense of tracking the state and decisions of every other firm. Based on the law of large numbers, firms only react to the average variable of interest of other firms. In this sense, the interactions between firms are mean-field. Yet, each firm is still smart, as it correctly anticipates ${ }^{3}$ the collective behavior of all firms, and sets the price accordingly.

3. Information Structure. We assume complete information of the demand the firm faces. More precisely, demand is deterministic. Each firm is also cognizant of its production cost, the mean-field price (reflecting aggregate market prices), and the mean-field cumulative demand (reflecting agregate market cumulative demands). Mean-field price and mean-field cumulative demand summarize all relevant market information.

\footnotetext{
${ }^{3}$ Technically, modeling rational anticipation is achieved with the forward/backward structure of the meanfield game.
} 
4. Dynamic Demand and Cost. Disregarding inventory, the model accounts for diffusion effects on the demand-side and learning effects on the supply side. Each firm's demand depends on its own price and on its cumulative (past) demand (diffusion effect). It also depends on the other firms through the mean-field price, and mean-field cumulative demand. The unit production cost of each firm decreases with its experience, approximated by its own cumulative demand/production (learning effect). While diffusion is related to aggregate firms, experience is tied to a single firm.

5. Objective Function. Each firm sells a unique product over an infinite planning horizon. The dynamic pricing policy of each firm maximizes the intertemporal profit, taking into account the evolution of demand. The discount rate affects firm's dynamic pricing policies. For mathematical tractability, all firms share the same demand and cost structure, although they differ in the initial cumulative demand. This heterogeneity in firms' initial experience characterizes different pricing schemes.

6. Equilibrium Concept. We model a competitive market, in which each firm takes profitmaximizing decisions over time. In this market, each firm sets its dynamic pricing policy, while believing that all other firms behave in the same way. Recall that the number of firms is infinite and these optimize according to the mean-field price and mean-field cumulative demand. Thus, the strategic behavior of firms can be modeled with a MFG framework. The resulting outcome is a MFG equilibrium, that is, a Nash equilibrium for an infinite number of players.

\section{Model Formulation}

We first introduce some notations. Then we describe the optimization problem of a single firm and the MFG equilibrium.

\subsection{Notations}

We consider a setting with continuous time $t$ and infinite horizon. Assuming away inventory, demand equals sales, which equal production.

Cumulative Demand of a Firm, State Variable. For a given firm, the cumulative demand at time $t$ is denoted $x_{t}$ (with $x_{0}$ reflecting the firm's initial experience). When prior conditions concerning cumulative demand $y$ at a previous time $\tau(\tau \leq t)$ need to be specified, we use the notation $x_{t}^{\tau, y}$ instead of $x_{t}$ (i.e., referring to the cumulative demand at time $t$ of the firm that faced the cumulative demand $y$ at the prior time $\tau$ ).

Mean-Field Cumulative Demand. At time $t=0$, firms differ in their initial cumulative demand $x_{0}$. The firms are indexed by $\omega \in \Omega$; the initial cumulative demand $x_{0}$ is thus a function of $\omega$ described as a (positive) random variable $X_{0}:(\Omega, \mathscr{F}, \mathbb{P}) \rightarrow \mathbb{R}_{+}$. Similarly, 
$X_{t}$ is the random variable of cumulative demands $x_{t}$ at time $t$. One can associate to $X_{t}$ its probability law $\mu_{t}(x)$ on $\mathbb{R}_{+}$and its average $\bar{X}_{t}=\mathbb{E}\left[X_{t}\right]$.

Remark 3.1. The model assumes the existence of an infinity of firms. In order to have something meaningful for the demand addressed to each firm, we must also assume the existence of an infinity of consumers. Otherwise, as any firm is negligible with respect to the sum of all others, the sales of any firm would be zero. The total market (reflected by the total units to sell) is therefore considered infinite, as well as the total profit at the market level. However, each firm makes a finite profit and sells a finite number of units. Formally, this implies $\bar{X}_{t} \leq N$, where $N$ is defined as the total units to sell on the market divided by the total number of firms. The parameter $N$ reflects the average market size (or maximum mean units) per firm. Note that a particular firm may sell more than $N$ units; inequality $\bar{X}_{t} \leq N$ is only true in average.

Price of a Firm, Control Variable. The pricing strategy of a firm consists in setting, at each time $t$, the price $p_{t}$ for the product.

Mean-Field Price. At time $t$, firms may charge different prices for the product. The observed price of firms $P_{t}^{M F}$ is a random variable $\omega \mapsto P_{t}^{M F}(\omega)$. Note that, for a given firm $\omega$ at time $t$, the notations distinguish between the observed price $P_{t}^{M F}(\omega)$ and the price set by the firm $p_{t}(\omega)$. Although $p_{t}(\omega)$ may differ from $P_{t}^{M F}(\omega)$, the two prices are equal at equilibrium.

Current demand. The firm's (current) demand at time $t, d x_{t} / d t$, depends on its situation, but also on the market conditions summarizing the behavior of the other firms. The firm's situation is characterized by (i) its cumulative demand $x_{t}$ (state variable), and (ii) its product price $p_{t}$ (control variable). Market conditions at time $t$ are reflected by: (i) the mean-field distribution of the cumulative demands of all firms captured through the average (mean-field) cumulative demand $\bar{X}_{t}{ }^{4}$, and (ii) the mean-field distribution of the market prices captured in the model through the average (mean-field) price $\bar{P}_{t}=\mathbb{E}\left[P_{t}^{M F}\right]$. Formally, demand at $t$ writes

$$
\frac{d x_{t}}{d t}=h\left(x_{t}, p_{t} ; \bar{X}_{t}, \bar{P}_{t}\right)
$$

Cost of Production. Accounting for the experience effect, a firm's unit production cost $c(x)$ depends on its total past demand, which equalizes cumulative production. The unit cost may be constant or decreasing. We consider the functional form

$$
c(x)=c_{1}\left(\frac{\bar{X}_{0}}{x}\right)^{\alpha},
$$

with the learning parameter $0<\alpha<1$.

\footnotetext{
${ }^{4} \mathrm{~A}$ more general formulation of the type $\frac{d x_{t}}{d t}=h\left(x_{t}, p_{t} ; \mu_{t}, P_{t}^{M F}\right)$ is possible when the firms have more information on the mean-field distributions of cumulative demands and prices.
} 
Intertemporal Profit, Discounted Payoff. Let $r \geq 0$ be the discount factor. The intertemporal profit (or present value of the profit stream over the planning period) of a firm with dynamic pricing $p_{t}$ starting at time $\tau$ from cumulative demand $y$ writes:

$$
\Pi\left(\tau, y, p ; \bar{X}_{t}, \bar{P}_{t}\right)=\int_{\tau}^{\infty} e^{-r t}\left\{p_{t}-c\left(x_{t}\right)\right\} \frac{d x_{t}}{d t} d t=\int_{\tau}^{\infty} e^{-r t}\left\{p_{t}-c\left(x_{t}\right)\right\} h\left(x_{t}, p_{t} ; \bar{X}_{t}, \bar{P}_{t}\right) d t,
$$

where we abuse the notation by writing $x_{t}$ for $x_{t}^{\tau, y}$. Often, we will omit $\bar{X}_{t}$ and $\bar{P}_{t}$ and only write $\Pi(\tau, y, p)$.

\subsection{Model}

The Firm's Problem. The optimization problem of each single firm is to maximize the intertemporal profit (3.3) over the planning period $[\tau, \infty)$ by setting at each time $t \geq \tau$ the price $p_{t}$, while taking into account the demand dynamics (3.1).

Optimal Profit. The optimal profit is the largest profit available among all admissible pricing rules:

$$
\Pi^{*}(\tau, y)=\sup \left\{\Pi(\tau, y, p) \mid p:\left[\tau, \infty\left[\rightarrow \mathbb{R}_{+}, \text {measurable, } p_{t} \geq 0, \forall t \geq 0\right\} .\right.\right.
$$

If it exists, an optimal price $p_{t}^{*}$ is a price that satisfies $\Pi^{*}(\tau, y)=\Pi\left(\tau, y, p^{*}\right)$. Note that $p^{*}$ is not necessarily unique.

MFG Equilibrium. To distinguish between optimal quantities and equilibrium quantities, we maintain the superscript notation ${ }^{*}$ for the first and employ the notation ${ }^{\dagger}$ for the latter. With these notations, an MFG equilibrium is reached when:

$$
\Pi\left(t, X_{t}^{\dagger}, P^{\dagger} ; \bar{X}_{t}^{\dagger}, \bar{P}_{t}^{\dagger}\right) \geq \Pi\left(t, X_{t}, p ; \bar{X}_{t}^{\dagger}, \bar{P}^{\dagger}\right), \forall t \geq 0, \forall p, X \text { (compatible according to }
$$

Remark 3.2. A game implies several players, yet, our game specification disregards the number of players. Indeed, we analyze here a limiting regime with an infinite number of firms. The resulting mean field equilibrium is defined regardless of a finite number of firms (in mathematical terms $\mathbb{P}\left(\left\{\omega_{1}, \omega_{2}, \ldots, \omega_{l}\right\}\right)=0, \forall \omega_{1}, \omega_{2}, \ldots, \omega_{l} \in \Omega$ and $\forall l \in \mathbb{N}$ ). In addition, with numerous firms, the fluctuations of cumulative demand and product price are expected to "average out" (see Adlakha et al. 2013, Subsection 2.2 for a discussion). Consequently, each firm may ignore the specific cumulative demand and price of every other particular firm. Instead, each firm simply sets its price based on the distribution $\mu_{t}$ of the cumulative demand $X_{t}$ of all other firms and on the observed market prices $P_{t}^{M F}$.

\section{Model Resolution}

To get economic insights, we now solve the problem by specifying the function $h$ expressed in (3.1). We consider the particular case where each firm's demand at $t$ depends on multiplica- 
tively separable functions of the market conditions $\left(\bar{X}_{t}\right.$ and $\left.\bar{P}_{t}\right)$ and the price set by the firm $\left(p_{t}\right)$. Demand (3.1) becomes

$$
\frac{d x_{t}}{d t}=f\left(\bar{X}_{t}, \bar{P}_{t}\right) g\left(p_{t}\right)
$$

with suitable hypotheses on $f$ and $g$, which will be made precise later.

The parametric examples $f\left(\bar{X}_{t}, \bar{P}_{t}\right)=\left(a+b \bar{X}_{t}\right)\left(N-\bar{X}_{t}\right) e^{k \bar{P}_{t}}$ and $g(p)=e^{-(d+k) p}$ yield

$$
\frac{d x_{t}}{d t}=\left(a+b \bar{X}_{t}\right)\left(N-\bar{X}_{t}\right) e^{-d p_{t}-k\left(p_{t}-\bar{P}_{t}\right)}
$$

where innovation $a>0$, imitation $b>0$, average market per firm $N>0$, price sensitivity $d>0$, and differential-price sensitivity $k>0$.

Remark 4.1. The market is assumed competitive, and the model does not allow market power for firms. However, if some consumers were more likely to buy from a given firm because of its cumulative sales (understood, for instance, as the installed base for network industries), then (4.1) may be generalized to $\frac{d x_{t}}{d t}=f\left(\bar{X}_{t}, \bar{P}_{t}, x_{t}\right) g\left(p_{t}\right)$. This generalization would call for an alternative treatment, left for future work.

For a firm starting at time $\tau$ from sales $y$ with the demand function (4.1), the intertemporal profit (3.3) writes

$$
\Pi(\tau, y, p)=\int_{\tau}^{\infty} e^{-r t}\left\{p_{t}-c\left(x_{t}\right)\right\} f\left(\bar{X}_{t}, \bar{P}_{t}\right) g\left(p_{t}\right) d t
$$

with $x_{t}^{\tau, y}$ being the precise meaning of $x_{t}$.

\section{$5 \quad$ Theoretical Results}

\subsection{Dynamic Pricing over Time}

We now consider a given firm and investigate its dynamic pricing strategy over time. The firm shares the same pricing rules as other firms. However, it may differ from other firms as it may not share the same initial cumulative demand $x_{0}$.

Assuming $f, g \geq 0$, we formulate the modeling hypotheses followed by some explanations.

(H1) $c^{\prime}\left(x_{t}\right) \leq 0$ for all $x_{t}>0$.

Hypothesis (H1) deals with the experience effect. It states that the unit production cost does not increase with firm experience.

(H2) $g^{\prime}(p)<0$ for all $p>0$.

Hypothesis (H2) deals with the impact of price on demand. It claims that firm's demand decreases with the increase in product price. 
(H3) $2\left(g^{\prime}(p)\right)^{2}>g(p) g^{\prime \prime}(p)$ for all $p>0$.

Hypothesis (H3) is a technical assumption frequent in dynamic pricing literature, required for profit optimization (see Remark 5.1).

(H4) $\lim _{p \rightarrow \infty} p g(p)=0$ (thus, in particular, $\lim _{p \rightarrow \infty} g(p)=0$ and $\lim _{p \rightarrow \infty} p+\frac{g(p)}{g^{\prime}(p)}=\infty$ ).

Hypothesis (H4) eliminates the possibility for firms to make infinite profit by selling zero products at an infinite price (as a limit process for $p \rightarrow \infty$ ).

(H5) $\bar{X}_{t}, \bar{P}_{t}$ and $Z_{\bar{X}_{t}, \bar{P}_{t}}=\int_{0}^{\infty} e^{-r t} f\left(\bar{X}_{t}, \bar{P}_{t}\right) d t$ are all bounded.

Hypothesis (H5) rules out the possibility for firms to earn infinite profits. We will show later that this assumption is always satisfied at equilibrium. Where there is no ambiguity, we write $Z$ instead of $Z_{\bar{X}_{t}, \bar{P}_{t}}$.

Remark 5.1. Hypotheses similar to (H3) also appear in Clarke et al. (1982, page 522, Equation (17)), in Kalish (1983, pages 140-143, Equations (5), (8), and (10)), in Dockner and Jørgensen (1988, page 324, Equation (3.2.3)), in Chen and Jain (1992, page 602, Equation (3.10)), and in Raman and Chatterjee (1995, page 147, Equation (7)). In these optimal control models, the hypothesis originates from the strict concavity of the Hamiltonian with respect to price (i.e., the control variable). Assumed to obtain a unique solution (provided its existence), this second order condition means that the marginal revenue is a non-decreasing function of price. Examples of demand functions satisfying (H3) include the exponential function $g(p)=e^{-d p}$, the linear function $g(p)=N-d p$, and the isoelastic function $g(p)=\frac{G}{p^{\gamma}}$, with $\gamma>1$ and $G>0$.

Theorem 5.2. Suppose that the average (mean-field) cumulative demand $\bar{X}_{t}$ and average (mean-field) price $\bar{P}_{t}$ are given and known to the firm and that (H1)-(H5) hold. Then:

1. If $\Pi^{*}(t, x)$ is differentiable everywhere, firm's optimal price $p_{t}^{*}$ is unique.

2. For $r>0$ the optimal price $p_{t}^{*}$ decreases monotonically over time.

3. For $r=0$ the optimal price $p_{t}^{*}$ is constant over time.

4. For any $t$, the optimal profit $\Pi^{*}(t, x)$ increases with respect to $x$ and $\lim _{x \rightarrow \infty} \Pi^{*}(t, x)<$ $\infty$.

Proof. The proof of Theorem 5.2 is given in Appendix A.

If the firm's demand only depends on one control variable, namely price, and the unit production cost decreases with experience, then Theorem 5.2 makes several claims. It first derives uniqueness conditions for the optimal pricing strategy. More precisely, when the optimal profit is differentiable everywhere, the optimal price is unique. Further, in case of a positive discount, the optimal price decreases monotonically throughout the planning period, 
while in case of no discount, the optimal price is constant over time. These theoretical results concerning dynamic pricing on competitive markets replicate prior findings in monopolistic (Kalish, 1983, Theorem 1, page 141) and oligopolistic markets (Dockner and Jørgensen, 1988, Theorem 1, page 324). The replication of results to competition is similar to that stressed in Yang and Xia (2013). In line with previous research based on the structural properties of the demand and cost function, Theorem 5.2 applies for arbitrary demand specification of (4.1). While the claim of declining prices in the competitive market matches the monopolistic and oligopolistic markets, a qualitative difference in optimal pricing exists between such markets. In competitive markets, firms enjoy no market power, and must follow the market price. Conversely, in monopolistic and oligopolistic markets, firms benefit from market power, which gives them some freedom in setting prices. In other words, firms are price takers in the competitive market, opposing price makers in the monopolistic and oligopolistic markets. Note that our results can be used as guidelines for competitive markets, but also to some extent, for less competitive markets.

Prior dynamic pricing literature does not investigate the role of initial advantage on firm situation and behavior. The MFG framework used here enables addressing this concern. Point 4 of Theorem 5.2 reveals that the optimal profit increases with the initial cumulative demand, stressing the importance of the initial advantage. Indeed, a firm with larger initial cumulative demand starts the planning period with a lower production cost. This firm can then always make pricing decisions at least as beneficial as a firm with a lower initial advantage. That is to say, the initial advantage persists over time. Note that because experience cannot decrease the unit production cost below zero, the optimal profit remains bounded even for arbitrary large initial cumulative demand.

Our analytic results reconcile the theoretical reflection on the role of past sales (or cumulative production) in prior research and the empirical evidence of declining price patterns. Krishnan et al. (1999, p. 1650) best describe the opposition between these ideas, "the research predominantly suggests that the optimal price path should be largely based on the sales growth pattern. However, in the real world we rarely find new products that have this pricing pattern. We observe either a monotonically declining pattern or an increase-decrease pricing pattern that does not seem close to the sales path." Thus, our work provides theoretical support for empirical observations of declining pricing paths.

Remark 5.3. The proof of Theorem 5.2 uses the property that its results hold if an exogenous temporal effect-modeled by a time function, say $l(t)$-affects demand in a multiplicative way. Consequently, the (qualitative) results of Theorem 5.2 hold if demand (4.1) writes $\frac{d x_{t}}{d t}=$ $f\left(\bar{X}_{t}, \bar{P}_{t}\right) g\left(p_{t}\right) l(t)$, where the temporal effect $l(t)$ accounts for a product life cycle effect, such as advertising, fashion, quality improvement, and the like. 


\subsection{Dynamic Pricing Across Firms}

In this section, we analyze the dynamic pricing policies across firms. For tractability, we consider the non-discounted case $r=0$ where firms behave as far-sighted players (see Remark 5.5 for the discounted case $r>0)$. To obtain finite sales for all firms, we introduce the additional hypothesis (H6) (consequence of Remark 3.1):

(H6) For any $v \geq 0$, the equation $\frac{d y(t)}{d t}=f(y, v), y(0)=\bar{X}_{0}$ is such that $\lim _{t \rightarrow \infty} y(t)=N$.

This technical assumption about the function $f$ in equation (4.1) imposes that, irrespective of the mean price, the market is saturated when time tends to the (infinite) planning horizon.

Note that (H6) is satisfied, for instance, with the parametric demand function (4.2). This hypothesis, used to prove the existence of an equilibrium, may be weakened.

Theorem 5.4. Suppose $f, g \geq 0$ fulfill hypotheses (H1)-(H4) and (H6). Then:

1. A unique equilibrium (in the sense of Equation (3.5)) exists.

2. Denoting $X_{t}^{\dagger}$ and $P_{t}^{\dagger}$ the random variables corresponding to equilibrium cumulative demand and price respectively, the following results hold:

(a) The average equilibrium price $\bar{P}_{t}^{\dagger}$ is constant over time.

(b) The variance of cumulative demand $\mathbb{V}\left(X_{t}^{\dagger}\right)$ and the variance of past cumulative demand $\mathbb{V}\left(X_{t}^{\dagger}-X_{0}\right)$ increase over time, while the variance of future cumulative demand $\mathbb{V}\left(X_{\infty}^{\dagger}-X_{t}^{\dagger}\right)$ decreases over time.

(c) The variance of past cumulative revenue $\mathbb{V}\left[\int_{0}^{t} P_{s}^{\dagger} \dot{X}_{s}^{\dagger} d s\right]$ increases over time and the corresponding Lorenz curve and Gini coefficient hold constant. The variance of future cumulative revenue $\mathbb{V}\left[\int_{t}^{\infty} P_{s}^{\dagger} \dot{X}_{s}^{\dagger} d s\right]$ decreases over time and the corresponding Lorenz curve and Gini coefficient remain constant.

(d) The Gini coefficient $G_{X_{t}^{\dagger}}$ of $X_{t}^{\dagger}$ is monotonic with respect to time. In particular, $G_{X_{t}^{\dagger}}$ increases when $G_{X_{0}^{\dagger}} \leq G_{g\left(P_{0}^{\dagger}\right)}$ and decreases when $G_{X_{0}^{\dagger}}>G_{g\left(P_{0}^{\dagger}\right)}$.

Proof. The proof of Theorem 5.4 is given in Appendix B.

Completing the classic results on oligopoly markets, the MFG approach allows to study market evolution in terms of distributions, showing the evolution (decrease of increase) of differences between firms over time. Theorem 5.4 reveals both existence and uniqueness results for the pricing policy of far-sighted firms in competitive markets (point 1). Further, at the equilibrium, the average price $\bar{P}_{t}$ remains constant over the planning horizon (point $2 \mathrm{a}$ ). Yet, each firm does not charge the average price. Instead, each firm sets its own (constant over 
time) optimal price, which depends on its initial cumulative demand $x_{0}$. Due to differences in the initial cumulative demand and thus in the optimal price, cumulative demand depends on each firm. More precisely, Theorem 5.4 claims that the variance of current and past cumulative demand increases with time (point $2 \mathrm{~b}$ ), translating into greater variance of past cumulative revenue (point 2c). Yet, the inequality in the distribution of $X_{t}^{\dagger}$ must not strengthen over the planning horizon. Indeed, the Gini coefficient of $X_{t}^{\dagger}$ is either monotonically increasing or decreasing (point 2d), depending on the dispersion of the initial cumulative demand and on the dispersion of the initial price.

With respect to extant literature on pricing policies over time, Theorem 5.4 informs on pricing policies across firms. In addition, the mean-field setup enables addressing the impact of differences in initial conditions on firm behavior. Theorem 5.4 explains the following market characteristic: firms (1) operating in a competitive market (no single firm enjoys market power), and (2) sharing the same optimizing rules, but (3) starting from a different initial advantage, set different price for the same product.

Remark 5.5. Equations (A.5)-(A.6) and (A.7) from Appendix A can be used to define an equilibrium for $r>0$. The technical considerations are more complicated, but not out of reach. In addition, a more powerful fixed point theorem is required (for instance the KakutaniGlicksberg-Fan or Tychonoff's fixed point theorem, as in Yang and Xia (2013)).

Remark 5.6. More general models such as $x_{t}^{\prime}=f\left(\bar{X}_{t}, \bar{P}_{t}, p\right)$ will no longer have the same $Z$ (defined in (H5)) for all $x_{0}$ and, thus, Brower's theorem can no longer be used. More sophisticated fixed point theorems are required, see Remark 5.5.

\section{$6 \quad$ Numerical Results}

This section presents the numerical simulations to illustrate the theoretical results in Section 5 and further explores the properties of the equilibrium.

\subsection{Parameters}

The parameters are as follows:

- The discount factor is null $r=0$, implying that firms are far-sighted.

- The initial distribution $\mu_{0}$ for firms' cumulative demand is a log-normal distribution ${ }^{5}$ of parameters mean -3 and standard deviation $\sigma=1 / 10$. This implies $\bar{X}_{0}=0.05$ and $\mathbb{V}\left(X_{0}\right)=2.5 e-05$. Figure 1 (left) plots this distribution.

- Functions $f, g$ are as in equation (4.2) with: $N=1, d=0.4, k=0.2, a=0.2, b=0.9$.

\footnotetext{
${ }^{5}$ A log-normal (or Gibrat) distribution accounts for the multiplicative product of numerous independent and identically-distributed variables, which are additive on a log scale.
} 
- The cost function is as in equation (3.2) with: $c_{1}=1.5, \alpha=0.4$.

In line with Xie and Sirbu (1995, Equation (5.1), p. 918), firms differ only in the initial conditions in cumulative demand, translating into different levels of production experience. In turn, experience affects the unit production cost. For simplicity, as in Kiesling et al. (2012, Figure 1, p. 187), the average market per firm $N$ is normalized to 1 . With this normalization and $\bar{X}_{0}=0.05$, the initial market penetration represents $5 \%$ of the market.

In their seminal article on numerical simulations of dynamic pricing, Robinson and Lakhani (1975, Equations (13) and (14)) consider a demand price sensitivity $d=0.35$. Parameter $d=0.4$ is slightly higher, but the differential price parameter $k=0.2$ lowers its effect. There is large agreement that $a<b$ (Sultan et al., 1990; Kiesling et al., 2012; Jiang and Jain, 2012), which our parameters verify. Numerical simulations use a range from $0.3<a+b<0.5$ (Kiesling et al., 2012, Figure 1, p. 187) and empirical estimates for cellular phones observe $0.2<a+b<0.4$ (Jiang and Jain, 2012, Table 3, p. 1893). These values match the range of "typical" values found in the review of Sultan et al. (1990, Table 3, p. 75), reporting estimates for room air conditioners such that $a<b$ and $0.3<a+b<1.1$. To render the numerical simulations visually clearer, we take large parameters with $a+b=1.1$.

The cost parameter $c_{1}=1.5$ falls within the range $[1,3]$ used by Xie and Sirbu (1995, Table 1, p. 919). Following Robinson and Lakhani (1975, Equation (15), p. 1118), the cost learning parameter is $\alpha=0.4$. That is, the experience curve makes the unit production cost decline by $25 \%$ each time the cumulated demand doubles.

\subsection{Market Equilibrium}

To compute the equilibrium, 40,000 independent samples are drawn from the distribution specified by $\mu_{0}$. Then, an iterative procedure computing the fixed point of the mapping ${ }^{6}$ $\mathscr{E}$ is performed. This yields the equilibrium price $p^{\dagger}(\cdot)$, plotted in Figure 1 (middle) (with the corresponding equilibrium value $\left.Z^{\dagger}=58.26\right)$. The intertemporal $\operatorname{profit}^{7} \Pi^{\dagger}\left(x_{0}\right)$ can be computed and is pictured in the right panel of Figure 1.

At the beginning of the planning horizon, firm's experience (reflected by $x_{0}$ ) determines the unit production cost $c\left(x_{0}\right)$, which affects firm's price and profit. Figure 1 (middle) illustrates that price $p^{\dagger}\left(x_{0}\right)$ decreases with initial cumulative demand; higher experience is associated with lower prices. A discontinuity in price is observed at $x_{0}^{s}=0.0529$. For $x_{0}<x_{0}^{s}$, the price declines up to 17.70. For $x_{0}>x_{0}^{s}$, the price is almost independent of the initial cumulative demand (stable at around 4.66). No firm charges the average market price $\bar{P}_{0}^{\dagger} \simeq 14.79$.

Figure 1 (right) shows that profit $\Pi^{\dagger}\left(x_{0}\right)$ increases with initial cumulative demand; higher experience allows for higher profit. A jump in the derivative of $\partial_{x_{0}} \Pi^{\dagger}\left(x_{0}\right)$ occurs at the same value $x_{0}^{s}$ as the discontinuity of $p^{\dagger}(\cdot)$. For $x_{0}>x_{0}^{s}$, the profit presents a clear increase with the initial cumulative demand, suggesting that differences in experience translate into strong

\footnotetext{
${ }^{6}$ The definition of the mapping $\mathscr{E}$ appears in the proof of point 1 from Theorem 5.4 in Appendix B.

${ }^{7}$ Recall that $\Pi^{\dagger}\left(x_{0}\right):=J^{*}\left(x_{0}, Z^{\dagger}\right)$, with $J^{*}$ defined in equation (B.6) in Appendix B.
} 

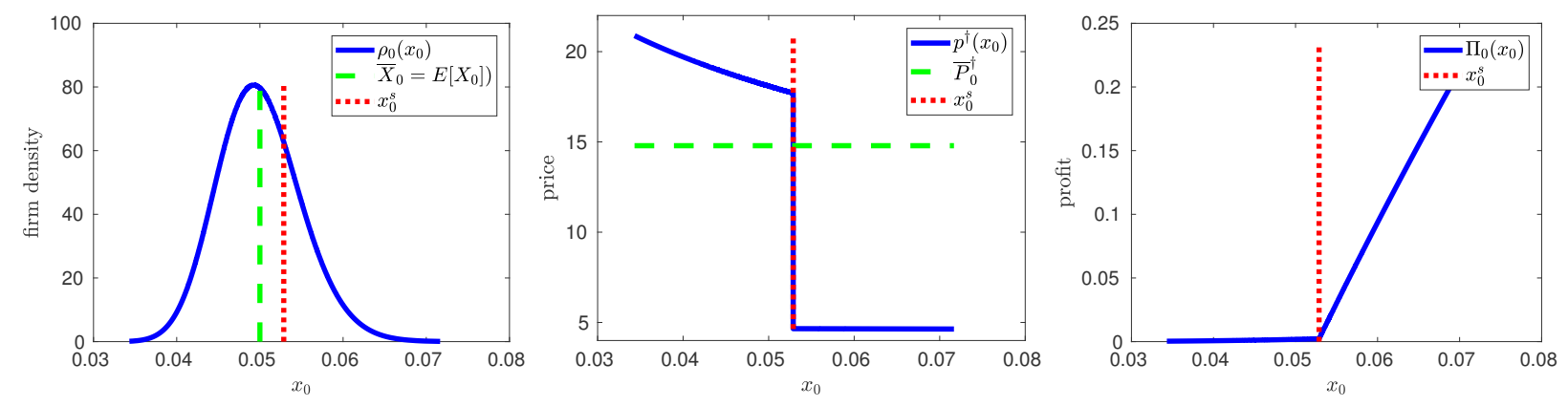

Figure 1: Left: Initial distribution $\mu_{0}$ of firms. Middle: $p^{\dagger}(\cdot)$. Right: $\Pi^{\dagger}(\cdot)$. Both $p^{\dagger}(\cdot)$ and $\Pi^{\dagger}(\cdot)$ lose regularity at the point $x_{0}^{s} \simeq 0.0529$. Although it is not possible to see the details at this scale, the profit $\Pi^{\dagger}\left(x_{0}\right)$ for values $x_{0}<x_{0}^{s}$ is never null (albeit small).

differences in profit. For $x_{0}<x_{0}^{s}$, differences in experience do not impact significantly the profit, which is almost constant and low, albeit positive. Numerical results inform that no firm exits the market: the profit of all firms is positive, even if very low for the majority.

\subsubsection{Experienced versus Inexperienced Firms}

The experience point $x_{0}^{s}$ generates a threshold with respect to both price $p^{\dagger}\left(x_{0}\right)$ (Figure 1 middle) and profit $\Pi^{\dagger}\left(x_{0}\right)$ (Figure 1 right). This suggests the co-existence of two groups of firms: those below and those above the experience threshold $x_{0}^{s}$. We further qualify these two groups as inexperienced and experienced firms, respectively. The asymmetry in the behavior of firms around the threshold $x_{0}^{s}$ informs that the two groups apply different pricing policies: inexperienced firms, representing $73.45 \%$ of the market, charge high prices that generate a low (but positive) profit; in contrast, experienced firms charge lower prices and derive higher profits. Pricing behavior allows to distinguish the two groups of firms more accurately than profit. Indeed, Figure 1 reveals, at the threshold, a discontinuity (a jump) in price (Figure 1 middle) opposing a continuity in profit (Figure 1 right): inexperienced firms set prices far above the average market price (i.e., 17.70 or higher), while experienced firms set prices far below the average market price (i.e., 4.66 or lower).

Figure 1 provides a statical picture of the situation of firms at $t=0$. Yet, the threshold effect identified in Figure 1 carries on over time. This idea is illustrated in Figure 2, which plots the distribution of the firms' cumulative demands (at the equilibrium) at different moments in time. The two groups of firms expand differently. Inexperienced firms do not evolve much: due to a large production cost, they have to charge more, consequently selling less. Therefore, the cumulative demand remains low, and the unit production cost holds almost constant over time. In contrast, experienced firms face increasing demand and detach from inexperienced firms. Due to a low production cost, they can charge less, maximizing profit by selling more. Thus, the cumulative demand increases over time, decreasing even more the unit production cost. The distinction between inexperienced and experienced firms subsists over time: no firm 
moves from one group to the other. Also, price, current demand, and profit appear relatively homogeneous for inexperienced firms and more heterogeneous for experienced firms. Highprofit firms benefit from a rent not tied to market power, but to initial experience.

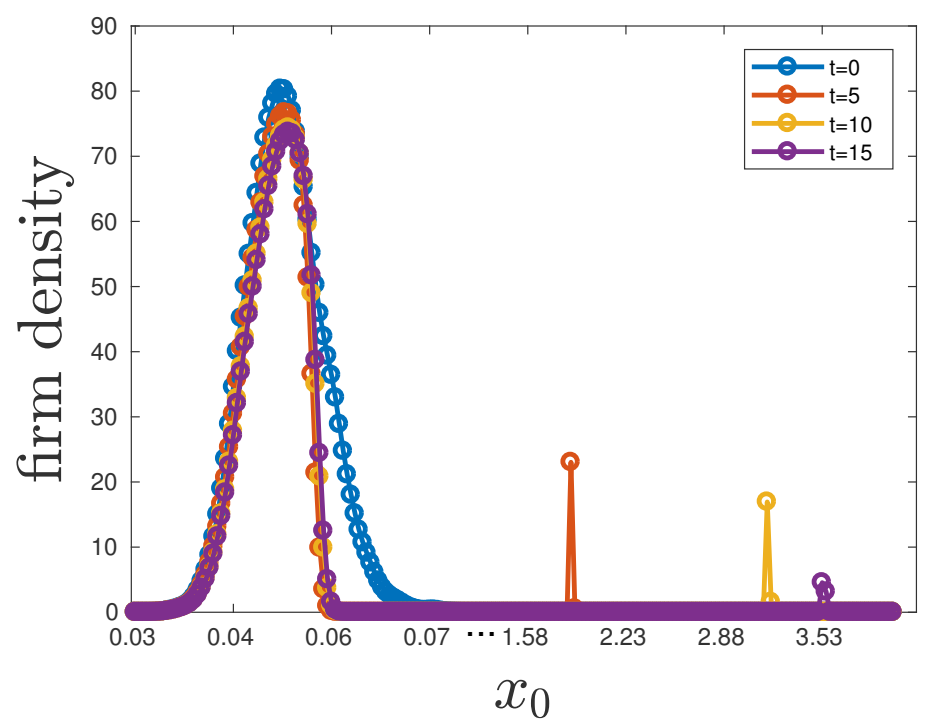

Figure 2: Cumulative demand $X_{t}$ at different moments in time.

\subsubsection{A Relative Experience Threshold}

The threshold $x_{0}^{s}$ separating inexperienced and experienced firms is relative, as opposed to absolute. Indeed, removing the inexperienced firms (below $x_{0}^{s}=0.0529$ ) from the market allows to compute a new equilibrium where, again, two separate groups of firms arise. We observe in the new equilibrium inexperienced firms with a high-price strategy and experienced firms with a low-price strategy. The experience threshold separating the two groups of firms almost doubles to 0.1072 , and the proportion of inexperienced firms slightly decreases to $64.20 \%$. Thus, after removing inexperienced firms from the market, a new set of inexperienced firms appears in a lower though still dominant proportion compared to the initial market. If, once again, the emergent group of inexperienced firms (below $x_{0}^{s}=0.1072$ ) is removed from the market, the threshold increases to $x_{0}^{s}=0.1556$ and the remaining firms still split between inexperienced (high price) firms (51.07\%) and experienced (low-price) firms (48.93\%). Alternatively, if in the initial distribution we remove the experienced firms from the market (firms above $x_{0}^{s}=0.0529$ ) and recompute the equilibrium, then the market again splits into two groups of inexperienced and experienced firms. The separating threshold decreases to $x_{0}^{s}=0.0340$ and the proportion of inexperienced firms changes to $76.69 \%$.

Thus, distinct groups of inexperienced (high-price) and experienced (low-price) firms arise in a competitive market. This finding is stable and dropping either the group of inexperienced 
or experienced firms does not change the matter: both groups of firms endogenously reappear on the market.

\subsection{Dispersion in Firms' Behavior over Time}

Figure 3 illustrates the findings from Theorem 5.4 in terms of variance. Consistent with point $2 \mathrm{~b}$ of Theorem 5.4, Figure 3 (left) shows that the variance of cumulative demand increases over time, reflecting an increase over time of the gap between experienced and inexperienced firms. Figure 3 (middle) illustrates that this effect translates into increased variance of past cumulative revenue over time (point 2c Theorem 5.4). Experienced firms succeed better than inexperienced firms in transforming their production experience into higher revenue. Figure 3 also informs that greater dispersion in cumulative demand (left) and in past cumulative revenue (middle) does not necessarily generate greater dispersion in future cumulative profit (right). The dispersion of future cumulative profit increases at the beginning of the planning horizon and falls at the end. This is consistent with the fact that while inexperienced firms face a regular increase in their profit, some experienced firms may accept losses at the beginning of the planning period in order to reduce their unit production cost, fully benefiting from the experience effect in later stages. At the end of the planning horizon, the dispersion is very low, but positive (some firms still have more profitable perspectives than others).
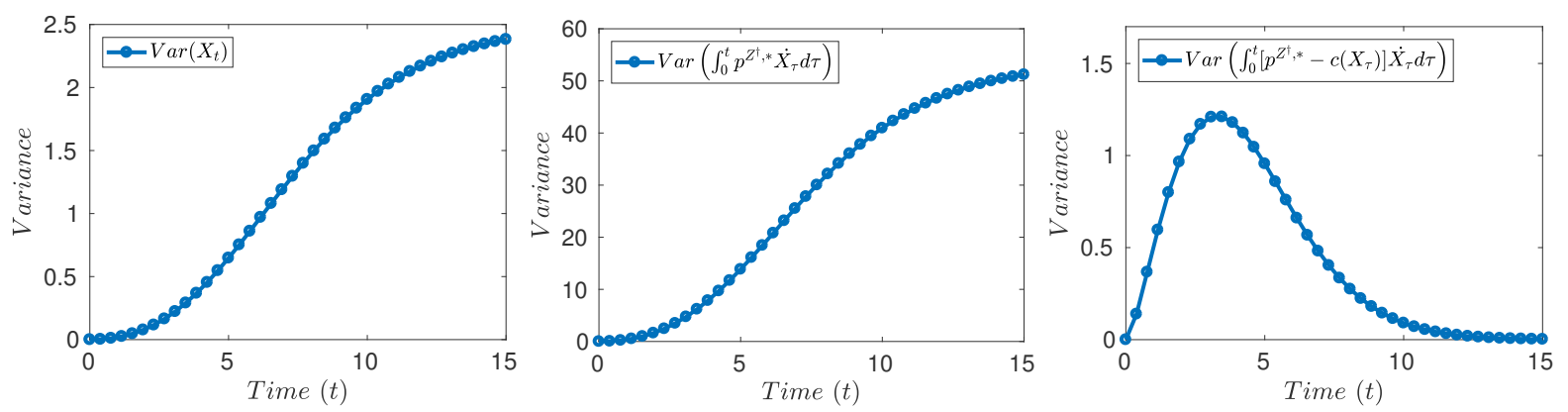

Figure 3: Variance of cumulative demand, past cumulative revenue, and future cumulative profit.

\subsection{Firm Heterogeneity}

The parameter $\sigma$ measures firms' heterogeneity with respect to initial cumulative demand, reflecting differences in production experience and in the unit production cost. A smaller $\sigma$ implies greater homogeneity among firms and a larger $\sigma$ greater heterogeneity. To explore the role of firm heterogeneity on competitive markets, we illustrate in Figure 4 the relation between $\sigma$ and several market characteristics. 

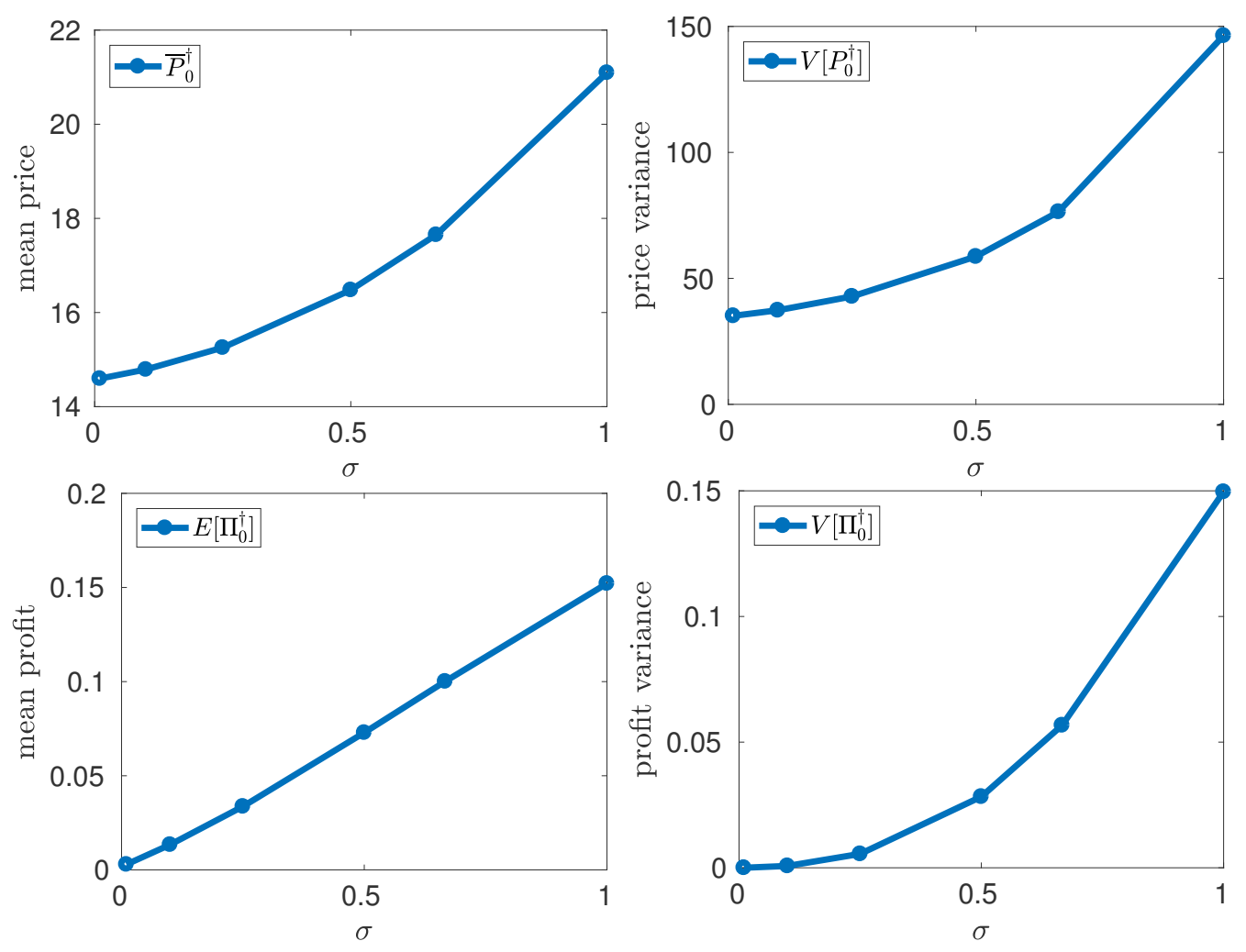

Figure 4: Firm heterogeneity $\sigma$ and statistics of price $P_{0}^{\dagger}$, price variance, profit $\Pi^{\dagger}\left(X_{0}\right)$ and profit variance.

Figure 4 (top left) illustrates the increase of the average market price $\bar{P}_{0}^{\dagger}$ with firm heterogeneity: prices are low for homogeneous firms and high for heterogeneous firms. Interestingly, buyers end up by paying for market heterogeneity in production experience. That is, consumers bear the cost of firms' heterogeneity by being charged more on average. For firms, higher prices turn into greater average profit $\Pi^{\dagger}\left(X_{0}\right)$ (Figure 4 bottom left). In particular, when firm heterogeneity increases ( $\sigma$ ranging from 0.01 to 1 ), the average market price increases by $45 \%$ and average profit from 0.0029 to 0.1521 . A small increase in average price goes hand in hand with a large improvement in average profit.

Figure 4 informs that firm heterogeneity also causes dispersion, both in terms of prices (top right) and profits (bottom right). These results highlight that differences in unit production cost between firms are enough to explain differences in price and profit. Such differences may not necessarily result from market power.

Overall, numerical simulations inform on the assessment of anti-competitive behavior. The findings highlight that, in a competitive market, differences in firm experience may explain heterogeneity in pricing, sales, and profit. Greater homogeneity in production costs, which plays a role in the supply-side and not the demand-side, is enough to lower prices and profits, 
and decrease price and profit dispersion. That is, (i) greater market power is not required to favor greater competition (in the sense of lower prices) and (ii) price dispersion does not necessarily signal price discrimination of consumers. Differences in price and profit may not be caused necessarily by market power but also by unit production costs. This finding suggests that the standard tools to monitor non-competitive behavior may fail in markets with intense competition. Higher mean and higher dispersion in price and profit, often taken as evidence of non-competitive practices, may simply reflect heterogeneity in production experience.

\section{Conclusion}

This article explored the dynamic pricing policy of new products in a competitive market with an infinity of non-atomic firms. A MFG framework characterized the optimal pricing schemes. Using Brower's fixed point theorem, we provided analytic results based on the structural (opposing parametric) properties of a general (non-linear) demand model. We proved the both existence and the uniqueness of the equilibrium. Results expose that, in a competitive market, price policies exhibit a constant or decreasing trend, which conforms prior findings on monopolistic and oligopolistic markets. We also investigated analytically the evolution of the dispersion of firms over time, found to be increasing for past cumulative demands and past revenues. The numerical investigations revealed that in a market with profit-maximizing far-sighted firms, two different groups of pricing strategies emerge endogenously depending on firm experience: more experienced firms use a low price strategy, while less experienced firms opt for a high price. Further, greater market heterogeneity in production experience causes greater average market price and profit, as well as higher dispersion.

The main market policy lesson derived from this research is that different pricing policies or profit streams are not enough to assess non-competitive behavior. Regarding price, there is no need of group collusion, dumping, limit pricing, and the like to observe different pricing schemes. Setting a higher or lower price may not reflect necessarily the market power of the firm. Greater dispersion in price, and thus in profit, conforms to the dynamics of competitive market, where firms differ in initial experience. Counter-intuitively, classic signals of anticompetitive behavior -based on price and profit- may prove inaccurate in competitive markets. This observation calls for a reexamination of the assessment on anticompetitive behaviors, enriching the debate on market regulation by administrative authorities.

Natural extensions of this work call for generalizing the homogeneous product case. Indeed, it would be desirable to analyze the cases of multi and differentiated products, which have received much attention in monopolistic and oligopolistic markets, but not in competitive markets. These extensions, although worthwhile, fall beyond the scope of this article. Nevertheless, the approach proposed here offers analytical and numerical tools that can be adapted to address such cases. Such adaptation would, in turn, foster deeper understanding of pricing phenomena in line with current managerial concerns. 


\section{Acknowledgements}

\section{A Appendix: Proof of Theorem 5.2}

\section{Proof. General considerations}

When the overall dynamics $\bar{X}_{t}$ and $\bar{P}_{t}$ is given, the situation enters, formally, the setting of Kalish (1983, bottom of page 141) and Clarke et al. (1982, Proposition 2 page 523). Consider the function

$$
p \in \mathbb{R}_{+} \mapsto \mathscr{L}(p)=p+\frac{g(p)}{g^{\prime}(p)} .
$$

Since $\mathscr{L}^{\prime}(p)=2-\frac{g(p) g^{\prime \prime}(p)}{\left(g^{\prime}(p)\right)^{2}}$ and hypothesis (H3) holds, we obtain $\mathscr{L}^{\prime}(p)>0$. $\mathscr{L}$ is thus a strictly increasing function. Its image is $\left[\frac{g(0)}{g^{\prime}(0)}, \infty\left[\right.\right.$. Since $g \geq 0$ and $g^{\prime}(p) \leq 0$ according to hypothesis (H2) we get $\frac{g(0)}{g^{\prime}(0)} \leq 0$. Thus, the image of $\mathscr{L}$ contains $\mathbb{R}_{+}$.

Proof of points 2 and 3

Same computations as in Kalish (1983) and Clarke et al. (1982) show that, for $r>0$, the optimum price $p^{*}$ satisfies :

$$
\frac{d p_{t}^{*}}{d t} \cdot \mathscr{L}^{\prime}\left(p_{t}^{*}\right)<0
$$

which together with hypothesis (H3) provide the point 2 of the conclusion.

For $r=0$, one obtains $\frac{d p_{t}^{*}}{d t} \mathscr{L}^{\prime}\left(p_{t}^{*}\right)=0$ thus $p_{t}^{*}$ is constant (point 3 of the conclusion).

Proof of point 1

We introduce the Hamiltonian

$$
H(t, p, \lambda)=(p-c(x)+\lambda) f\left(\bar{X}_{t}, \bar{P}_{t}\right) g(p) .
$$

The optimal price $p^{*}$ will maximize $H$ with respect to $p$ when $\lambda$ is the adjoint state $\lambda^{*}$; recalling that at the optimal solution, $\lambda^{*}(t)=\partial_{x} \Pi^{*}\left(t, x_{t}\right)$.

With the market conditions given, the maximization of (A.3) is related to the maximization of $p \mapsto(p-\beta) g(p)$ (with the particular case of interest $\beta=c(x)-\lambda$ ). Its derivative is $g(p)+(p-\beta) g^{\prime}(p)$ which is positive when $\beta \geq \mathscr{L}(p)$ or, equivalently, $\mathscr{L}^{-1}(\beta) \geq p$ (for $\beta$ in the domain of $\mathscr{L})$. Thus $(p-\beta) g(p)$ increases and then decreases; it has a unique maximum attained at $\mathscr{L}^{-1}(\beta)$. Going back to the maximization of equation (A.3) it results that the optimal price satisfies $p_{t}^{*}=\mathscr{L}^{-1}\left(c\left(x_{t}\right)-\lambda_{t}\right)=\mathscr{L}^{-1}\left(c\left(x_{t}\right)-\partial_{x} \Pi^{*}\left(t, x_{t}\right)\right)$, which shows that the optimal price is unique when $\Pi^{*}(t, x)$ is differentiable with respect to $x$ everywhere (and not necessarily so otherwise).

\section{Proof of point 4}

Intuitively the profit $\Pi^{*}$ is increasing with respect to $x$ because a firm with higher initial sales can use the same strategy as a firm with lower initial sales. but its cost will be lower, which allows for profit higher. The rigorous transcription of this idea is as follows: consider two firms with initial sales $x_{0}^{1}$ and $x_{0}^{2}$, with $x_{0}^{1} \leq x_{0}^{2}, p$ some pricing strategy as in (3.4) and 
$x_{t}^{\tau, x_{0}^{1}}$ and $x_{t}^{\tau, x_{0}^{2}}$ the sales of the firms using the strategy $p$. Then $\frac{d}{d t} x_{t}^{\tau, x_{0}^{2}}=\frac{d}{d t} x_{t}^{\tau, x_{0}^{1}}$ and thus $x_{t}^{\tau, x_{0}^{2}} \geq x_{t}^{\tau, x_{0}^{1}}$ for any $t \geq \tau$ which shows that $c\left(x_{t}^{\tau, x_{0}^{2}}\right) \leq c\left(x_{t}^{\tau, x_{0}^{1}}\right)$ for any $t \geq \tau$. But then, recalling definition (4.3), $\Pi\left(\tau, x_{0}^{2}, p\right) \geq \Pi\left(\tau, x_{0}^{1}, p\right)$. Taking now the supremum with respect to $p$ and using (3.4) it follows that

$$
\Pi^{*}\left(\tau, x_{0}^{2}\right)=\sup _{p} \Pi\left(\tau, x_{0}^{2}, p\right) \geq \sup _{p} \Pi\left(\tau, x_{0}^{1}, p\right)=\Pi^{*}\left(\tau, x_{0}^{1}\right),
$$

proving the first part of point 4 .

Denote $\mathscr{H}(\beta)=\max _{p \geq 0}(p-\beta) g(p)$. The optimal profit $\Pi^{*}$ is the unique viscosity solution (see Crandall and Lions (1983); Bressan and Piccoli (2007); Bardi and Capuzzo-Dolcetta (1997) for more details) of the following Hamilton-Jacobi-Bellman equation:

$$
\begin{aligned}
& \partial_{t} \Pi^{*}(t, x)+\mathscr{H}\left(c(x)-\partial_{x} \Pi^{*}(t, x)\right) f\left(\bar{X}_{t}, \bar{P}_{t}\right)-r \Pi^{*}(t, x)=0 \\
& \Pi^{*}(\infty, x)=0 .
\end{aligned}
$$

In addition, at any point $x_{t}$ where $\partial_{x} \Pi^{*}\left(t, x_{t}\right)$ exists the optimal price $p_{t}^{*}$ of a firm with cumulative sales $x_{t}$ at time $t$ only depends on $t$ and $x_{t}$ and satisfies :

$$
p_{t}^{*}=\mathscr{L}^{-1}\left(c\left(x_{t}\right)-\partial_{x} \Pi^{*}\left(t, x_{t}\right)\right) \leq \mathscr{L}^{-1}\left(c\left(x_{t}\right)\right) \leq \mathscr{L}^{-1}\left(c\left(x_{0}\right)\right) .
$$

Thus, hypothesis (H4) and (H5) allow bounding the profit for $x_{0} \rightarrow \infty$. For $x_{0} \rightarrow 0$ the optimal price can be unbound (since $c\left(x_{0}\right)$ may tend to $\infty$ for $x_{0} \rightarrow 0$ ) but the profit will certainly be finite (being inferior to any profit for fixed $x_{0}>0$ ), which proves the second part of point 4 of the conclusion.

\section{B Appendix: Proof of Theorem 5.4}

\section{Proof. General considerations}

Point 3 of Theorem 5.2 gives that for $r=0$, the optimal price $p^{*}$ of a representative firm is constant. Given $\bar{X}_{t}, \bar{P}_{t}$ (not necessarily at equilibrium) the (constant) optimal price $p^{*}$ of a firm maximizes the profit (obtained from equation (4.3) with $p$ constant using (4.1)) :

$$
\int_{0}^{\infty}\left(p-c\left(x_{t}\right)\right) \frac{d x_{t}}{d t} d t=p \cdot\left(x_{\infty}-x_{0}\right)-\left[I_{c}\left(x_{\infty}\right)-I_{c}\left(x_{0}\right)\right],
$$

where $I_{c}(\cdot)$ refers to a primitive of the cost function $c$; that is, $I_{c}^{\prime}(y)=c(y)$, for any $y>0$.

Using the definition of $Z=Z_{\bar{X}_{t}, \bar{P}_{t}}$, which is fixed and integrating (4.1) with respect to time, yields $x_{\infty}=x_{0}+Z g(p)$. To determine the optimal price $p^{*}=p^{Z, *}\left(x_{0}\right)$ that maximizes equation (B.1), we define the profit function $J$ :

$$
J\left(p ; x_{0}, Z\right):=p Z g(p)-\left[I_{c}\left(x_{0}+Z g(p)\right)-I_{c}\left(x_{0}\right)\right] .
$$


Note that $J$ is increasing with respect to $x_{0}$. By differentiating $J$ with respect to $p$ we obtain $Z\left(p^{*} g^{\prime}\left(p^{*}\right)+g\left(p^{*}\right)\right)=c\left(x_{0}+Z g\left(p^{*}\right)\right) Z g^{\prime}\left(p^{*}\right)$. Divide now both terms by $Z g^{\prime}\left(p^{*}\right)$, to obtain $p^{*}+g\left(p^{*}\right) / g^{\prime}\left(p^{*}\right)=c\left(x_{0}+Z g\left(p^{*}\right)\right)$, which can also be written as $\mathscr{L}\left(p^{*}\right)=c\left(x_{0}+Z g\left(p^{*}\right)\right)$. Hence, after inverting the function $\mathscr{L}$ and indicating the explicit dependence on $x_{0}$, we obtain:

$$
p^{Z, *}\left(x_{0}\right)=\mathscr{L}^{-1}\left[c\left(x_{0}+Z g\left(p^{Z, *}\left(x_{0}\right)\right)\right)\right] \leq \mathscr{L}^{-1}\left(c\left(x_{0}\right)\right) .
$$

Note that at this time $p^{Z, *}\left(x_{0}\right)$ is not necessarily unique. However, we can show that $p^{Z, *}\left(x_{0}\right)$ is decreasing with respect to $x_{0}$; indeed consider $x_{0}^{1}<x_{0}^{2}$. The optimum price being constant means that we must have for any $t \geq 0$

$$
x_{t}^{x_{0}^{1}, *}<x_{t}^{x_{0}^{2}, *} .
$$

Otherwise, some point $\bar{t}$ exists with $x_{\bar{t}}^{x_{0}^{1}, *}=x_{\bar{t}}^{x_{0}^{2}, *}$ and thus a non-constant price can be constructed that is optimal for the firm starting in $x_{0}^{2}$. However, (B.4) implies $x_{\infty}^{x_{0}^{1}, *} \leq x_{\infty}^{x_{0}^{2}, *}$, which also implies

$$
p^{Z, *}\left(x_{0}^{1}\right)=\mathscr{L}^{-1}\left[c\left(x_{\infty}^{x_{0}^{1}, *}\right)\right] \geq \mathscr{L}^{-1}\left[c\left(x_{\infty}^{x_{0}^{2}, *}\right)\right]=p^{Z, *}\left(x_{0}^{2}\right) .
$$

A closer analysis reveals that $p^{Z, *}\left(x_{0}\right)$ is in fact strictly monotonic. Thus $p^{Z, *}\left(x_{0}\right)$ is strictly decreasing with respect to $x_{0}$, has left and right limits (which are optimums) and any other (optimal) value is between these limits.

On the other hand, the function

$$
\left(x_{0}, Z\right) \mapsto J^{*}\left(x_{0}, Z\right)=\sup _{p \geq 0} J\left(p, x_{0}, Z\right),
$$

is Lipschitz thus differentiable almost everywhere with respect to $x_{0}$; in any point of differentiability of $J^{*}\left(x_{0}, Z\right)$ we have that $p^{Z, *}\left(x_{0}\right)$ is uniquely given by (compare with formula $(\mathrm{A} .7)$ )

$$
p^{Z, *}\left(x_{0}\right)=\mathscr{L}^{-1}\left[c\left(x_{0}\right)-\partial_{x_{0}} J^{*}\left(x_{0}, Z\right)\right] .
$$

Let us now inquire about the variation of $p^{Z, *}\left(x_{0}\right)$ with respect to $Z$ : consider $Z_{2}>Z_{1}$ and the optimal dynamics $x_{t}^{x_{0}, *}$ of a firm starting in $x_{0}$; at time $t=0$ the price $p^{Z_{2}, *}\left(x_{0}\right)$ represents a maximum of the profit $J\left(x_{0}, Z_{2}=\int_{0}^{\infty} f\left(\bar{X}_{t}, \bar{P}_{t}\right) d t, p\right)$ with respect to $p$, while at a later time $t \geq 0$ it will also be an optimum of $J\left(x_{0}, \int_{t}^{\infty} f\left(\bar{X}_{t}, \bar{P}_{t}\right) d t, p\right)$. Choosing the time $t$ for which $Z_{1}=\int_{t}^{\infty} f\left(\bar{X}_{t}, \bar{P}_{t}\right) d t$ we obtain $p^{Z_{2}, *}\left(x_{0}\right)=p^{Z_{1}, *}\left(x_{t}^{x_{0}}\right)<p^{Z_{1}, *}\left(x_{0}\right)$, where we used the monotonicity of $p^{Z, *}\left(x_{0}\right)$ with respect to $x_{0}$; thus we obtain that $p^{Z, *}\left(x_{0}\right)$ is strictly decreasing with respect to $Z$.

In summary, $x_{0} \mapsto p^{Z, *}\left(x_{0}\right)$ is strictly decreasing; it is thus continuous with exception of at most countable set of points $x_{0}$. The same holds for $Z \mapsto p^{Z, *}\left(x_{0}\right)$.

To avoid lengthy technical developments, we will only present the reminder of the proof for the situation when $\mu_{0}$ admits a density $\rho_{0}\left(x_{0}\right)$ i.e., $\mu_{0}=\rho_{0}\left(x_{0}\right) d x_{0}$. 
When $\mu_{0}$ does not admit a density, then it may occur that for a set $A$ (with $\mu_{0}(A)>0$ ) of firms $\omega \in A$, the price $p$ is not necessarily unique, which means that two firms starting from the same $x_{0}$ can choose different prices. In mathematical terms $p$ depends not only on the cumulative sales $X_{0}(\omega)$ of the firm $\omega$ but also on the firm $\omega$ itself: $p=p\left(\omega, X_{0}(\omega)\right)$; in this case $\mathscr{E}(\cdot)$ is a set-valued map and the proof has to use more general fixed point theorems (see Remark 5.5).

Moreover, to avoid degenerate settings, we also assume that the cost function $c(\cdot)$ is always strictly positive.

\section{Proof of point $2 \mathrm{a}$}

Since each firm has constant optimal price, the equilibrium mean price $\bar{P}_{t}^{\dagger}$ will also be constant:

$$
\bar{P}_{t}^{\dagger}=\mathbb{E}\left[P_{t}^{\dagger}\right]=\mathbb{E}\left[P_{0}^{\dagger}\right]
$$

In addition, hypothesis (H6) ensures that $\bar{X}_{t}^{\dagger} \in[0, N]$ and $\bar{X}_{t}^{\dagger} \rightarrow N$ for $t \rightarrow \infty$.

\section{Proof of point 1}

The proof of existence relies on the remark that to each possible $\bar{X}_{t}$ and $\bar{P}_{t}$ a number $Z$ corresponds. We will find the $Z$ that gives an equilibrium. Note first that any equilibrium $Z$ can be bounded by some universal constant $Z_{\text {max }}^{\mu_{0}}:=\frac{N-\bar{X}_{0}}{\mathbb{E}\left[g\left(\mathscr{L}^{-1}\left(c\left(X_{0}\right)\right)\right)\right]}$; thus, it is enough to look for an equilibrium in the interval $\left[0, Z_{\text {max }}^{\mu_{0}}\right]$. Let $Z \in\left[0, Z_{\text {max }}^{\mu_{0}}\right]$; then one can associate to it the optimal price $p^{Z, *}\left(x_{0}\right)$ maximizing $J\left(p, x_{0}, Z\right)$ (with respect to $p$ ). This price is unique for almost all $x_{0}$; for $Z_{n} \rightarrow Z$ any accumulation point of $\left\{p^{Z_{n}, *}\left(x_{0}\right), n \geq 1\right\}$ is necessarily an optimum for $J\left(p, x_{0}, Z\right)$, thus by uniqueness $p^{Z, *}\left(x_{0}\right)=\lim _{n \rightarrow \infty} p^{Z_{n}, *}\left(x_{0}\right)$ a.e. in $x_{0}$. The mapping $Z \in\left[0, Z_{\text {max }}^{\mu_{0}}\right] \mapsto \mathscr{E}(Z)=\frac{N-\bar{X}_{0}}{\mathbb{E}\left[g\left(p^{Z, *}\left(X_{0}\right)\right)\right]} \in\left[0, Z_{\text {max }}^{\mu_{0}}\right]$ is thus well defined and continuous; it must have a fixed point (according to Brower's theorem) that corresponds to an equilibrium.

Also note that $\mathscr{E}(Z) \geq \frac{N-\bar{X}_{0}}{g(0)}>0$. The strict monotony of $p^{Z, *}\left(x_{0}\right)$ with respect to $Z$ implies that $\mathscr{E}(Z)$ is decreasing with respect to $Z$ and thus the fixed point is unique.

\section{Proof of point $2 \mathrm{~b}$}

Now take $Z^{\dagger}$ as the equilibrium value and $p^{\dagger}(\cdot)=p^{Z^{\dagger}, *}(\cdot)$; note that

$$
x_{t}^{\dagger, x_{0}}=x_{0}+g\left(p^{\dagger}\left(x_{0}\right)\right) \frac{\bar{X}_{t}^{\dagger}-\bar{X}_{0}}{\mathbb{E}\left[g\left(p^{\dagger}\left(X_{0}\right)\right)\right]}, X_{t}^{\dagger}=X_{0}+g\left(P_{0}^{\dagger}\right) \frac{\bar{X}_{t}^{\dagger}-\bar{X}_{0}}{\mathbb{E}\left[g\left(P_{0}^{\dagger}\right)\right]} .
$$

To prove that $\mathbb{V}\left(X_{t}\right)$ is increasing with respect to $t$, we will use an alternative formula for the variance of a random variable $Y$ :

$$
\mathbb{V}(Y)=\frac{1}{2}\left\{\int_{\Omega} \int_{\Omega}\left[\left(Y\left(\omega_{1}\right)-Y\left(\omega_{2}\right)\right]^{2} \mathbb{P}\left(d \omega_{1}\right) \mathbb{P}\left(d \omega_{2}\right)\right\} .\right.
$$

Or, formula (B.9) and the monotonicity of $p^{Z, *}\left(x_{0}\right)$ with respect to $x_{0}$ shows that for any $y_{1}=X_{0}\left(\omega_{1}\right), y_{2}=X_{0}\left(\omega_{1}\right) \geq 0$ :

$$
\left|X_{t}\left(\omega_{1}\right)-X_{t}\left(\omega_{2}\right)\right|=\left|x_{t}^{\dagger, y_{1}}-x_{t}^{\dagger, y_{2}}\right| \geq\left|y_{1}-y_{2}\right|=\left|X_{0}\left(\omega_{1}\right)-X_{0}\left(\omega_{2}\right)\right|,
$$


which implies, using (B.10), that $\mathbb{V}\left(X_{t}\right) \geq \mathbb{V}\left(X_{0}\right)$.

The other parts of the conclusion are a consequence of formula (B.9) and the monotony of $\bar{X}_{t}^{\dagger}-\bar{X}_{0}$ and $\bar{X}_{\infty}^{\dagger}-\bar{X}_{t}^{\dagger}$ respectively.

\section{Proof of point 2c}

We only analyze here the past cumulative revenue $\mathbb{V}\left[\int_{0}^{t} P_{s}^{\dagger} \dot{X}_{s}^{\dagger} d s\right]$, while the future cumulative revenue $\mathbb{V}\left[\int_{t}^{\infty} P_{s}^{\dagger} \dot{X}_{s}^{\dagger} d s\right]$ is left as an exercise for the reader.

Recalling that with firm's price being constant in time, the past cumulative revenue is simply $P_{0}^{\dagger}\left(X_{t}^{\dagger}-X_{0}\right)$; equation (B.9) shows that $\mathbb{V}\left(P_{0}^{\dagger}\left(X_{t}^{\dagger}-X_{0}\right)\right)=\mathbb{V}\left[P_{0}^{\dagger} g\left(P_{0}^{\dagger}\right)\right] \frac{\left(\bar{X}_{t}^{\dagger}-\bar{X}_{0}\right)^{2}}{\mathbb{E}\left[g\left(p^{\dagger}\left(X_{0}\right)\right)\right]^{2}}$ which is increasing because $\bar{X}_{t}^{\dagger}-\bar{X}_{0}$ is positive increasing. The conclusion on the Lorenz curve follows from the remark that cumulative revenue $P_{0}^{\dagger}\left(X_{t}^{\dagger}-X_{0}\right)$ is the product of a time-dependent real constant $\bar{X}_{t}^{\dagger}-\bar{X}_{0}$ and a time-independent random variable $\frac{P_{0}^{\dagger} g\left(P_{0}^{\dagger}\right)}{\mathbb{E}\left[g\left(P_{0}^{\dagger}\right)\right]}$.

\section{Proof of point 2d}

Recall that the Gini coefficient of a real variable $B$ is

$$
G_{B}=\frac{\int_{\Omega} \int_{\Omega}\left|B\left(\omega_{1}\right)-B\left(\omega_{2}\right)\right| \mathbb{P}\left(d \omega_{1}\right) \mathbb{P}\left(d \omega_{2}\right)}{2 \mathbb{E}[B]} .
$$

Thus

$$
\begin{aligned}
& G_{X_{t}^{\dagger}}=\frac{\int_{\Omega} \int_{\Omega}\left|X_{t}^{\dagger}\left(\omega_{1}\right)-X_{t}^{\dagger}\left(\omega_{2}\right)\right| \mathbb{P}\left(d \omega_{1}\right) \mathbb{P}\left(d \omega_{2}\right)}{2 \mathbb{E}\left[X_{t}\right]} \\
& =\frac{\int_{\Omega} \int_{\Omega}\left|X_{0}^{\dagger}\left(\omega_{1}\right)-X_{0}^{\dagger}\left(\omega_{2}\right)+\left[g\left(P_{0}^{\dagger}\left(\omega_{1}\right)\right)-g\left(P^{\dagger}\left(\omega_{2}\right)\right)\right] \frac{\bar{X}_{t}^{\dagger}-\bar{X}_{0}}{\mathbb{E}\left[g\left(P_{0}^{\dagger}\right)\right]}\right| \mathbb{P}\left(d \omega_{1}\right) \mathbb{P}\left(d \omega_{2}\right)}{2 \mathbb{E}\left[X_{t}\right]} .
\end{aligned}
$$

But since $y_{1}-y_{2}$ and $g\left(p^{\dagger}\left(y_{1}\right)\right)-g\left(p^{\dagger}\left(y_{2}\right)\right)$ have the same sign and $\frac{\bar{X}_{t}^{\dagger}-\bar{X}_{0}}{\mathbb{E}\left[g\left(P_{0}^{\dagger}\right)\right]}>0$ we obtain:

$$
\begin{aligned}
& \left|X_{0}^{\dagger}\left(\omega_{1}\right)-X_{0}^{\dagger}\left(\omega_{2}\right)+\left[g\left(P_{0}^{\dagger}\left(\omega_{1}\right)\right)-g\left(P^{\dagger}\left(\omega_{2}\right)\right)\right] \frac{\bar{X}_{t}^{\dagger}-\bar{X}_{0}}{\mathbb{E}\left[g\left(P_{0}^{\dagger}\right)\right]}\right|=\left|X_{0}^{\dagger}\left(\omega_{1}\right)-X_{0}^{\dagger}\left(\omega_{2}\right)\right| \\
& +\left|g\left(P_{0}^{\dagger}\left(\omega_{1}\right)\right)-g\left(P^{\dagger}\left(\omega_{2}\right)\right)\right| \frac{\bar{X}_{t}^{\dagger}-\bar{X}_{0}}{\mathbb{E}\left[g\left(P_{0}^{\dagger}\right)\right]}
\end{aligned}
$$

and after some computations,

$$
G_{X_{t}^{\dagger}}=\frac{\mathbb{E}\left[X_{0}\right]}{\mathbb{E}\left[X_{t}^{\dagger}\right]} G_{X_{0}}+\left(1-\frac{\mathbb{E}\left[X_{0}\right]}{\mathbb{E}\left[X_{t}^{\dagger}\right]}\right) G_{g\left(P_{0}^{\dagger}\right)},
$$

hence the conclusion follows. 


\section{References}

Adlakha, S. and R. Johari (2013a). Mean field equilibrium in dynamic games with strategic complementarities. Operations Research 61(4), 971-989.

Adlakha, S. and R. Johari (2013b). Mean field equilibrium in dynamic games with strategic complementarities. Operations Research 61(4), 971-989.

Adlakha, S., R. Johari, and G. Y. Weintraub (2013). Equilibria of dynamic games with many players: Existence, approximation, and market structure. Journal of Economic Theory 156 (March), 269-316.

Adlakha, S., R. Johari, and G. Y. Weintraub (2015). Equilibria of dynamic games with many players: Existence, approximation, and market structure. Journal of Economic Theory 156, 269-316.

Bardi, M. and I. Capuzzo-Dolcetta (1997). Optimal control and viscosity solutions of Hamilton-Jacobi-Bellman equations. Systems \& Control: Foundations \& Applications. Boston, MA: Birkhäuser Boston Inc. With appendices by Maurizio Falcone and Pierpaolo Soravia.

Bass, F. (1969). A new product growth for model consumer durables. Management Science 15(5), 215-227.

Bauso, D., H. Tembine, and T. Basar (2012). Robust mean field games with application to production of an exhaustible resource. In Robust Control Design, Volume 7, pp. 454-459.

Bensoussan, A., J. Frehse, P. Yam, et al. (2013). Mean Field Games and Mean Field Type Control Theory, Volume 101. Springer.

Bressan, A. and B. Piccoli (2007). Introduction to the mathematical theory of control, Volume 2 of AIMS Series on Applied Mathematics. American Institute of Mathematical Sciences (AIMS), Springfield, MO.

Carmona, R. and F. Delarue (2017-2018). Probabilistic Theory of Mean Field Games with Applications I-II. Springer International Publishing.

Carmona, R., J.-P. Fouque, and L.-H. Sun (2013). Mean field games and systemic risk. Available at SSRN 2307814.

Chan, P. and R. Sircar (2015). Bertrand and cournot mean field games. Applied Mathematics \& Optimization $71(3), 533-569$.

Chatterjee, R. (2009). Strategic pricing of new product and services. In V. Rao (Ed.), Handbook of Pricing Research in Marketing, pp. 169-215. MA: Edward Elgar Publishing. 
Chen, M. and Z.-L. Chen (2015). Recent developments in dynamic pricing research: Multiple products, competition, and limited demand information. Production and Operations Management 24(5), 704-731.

Chen, Y. and V. F. Farias (2013). Simple policies for dynamic pricing with imperfect forecasts. Operations Research 61(3), 612-624.

Chen, Y.-M. and D. C. Jain (1992). Dynamic monopoly pricing under a Poisson-type uncertain demand. Journal of Business 65(4), 593-614.

Chutani, A. and S. P. Sethi (2012). Optimal advertising and pricing in a dynamic durable goods supply chain. Journal of Optimization Theory and Applications 154(2), 615-643.

Clarke, F. H., M. N. Darrough, and J. M. Heineke (1982). Optimal pricing policy in the presence of experience effects. Journal of Business 55(4), 517-530.

Crandall, M. G. and P.-L. Lions (1983). Viscosity solutions of Hamilton-Jacobi equations. Trans. Amer. Math. Soc. 277(1), 1-42.

Danaher, P. J., B. G. Hardie, and W. P. Putsis Jr (2001). Marketing-mix variables and the diffusion of successive generations of a technological innovation. Journal of Marketing Research 38(4), 501-514.

Den Boer, A. V. (2015a). Dynamic pricing and learning: Historical origins, current research, and new directions. Surveys in Operations Research and Management Science 20(1), 1-18.

Den Boer, A. V. (2015b). Tracking the market: Dynamic pricing and learning in a changing environment. European Journal of Operational Research 247(3), 914-927.

Dockner, E. and S. Jørgensen (1988). Optimal pricing strategies for new products in dynamic oligopolies. Marketing Science 7(4), 315-334.

Dockner, E., S. Jorgenssen, V. N. Long, and S. Gerhard (2000). Differential Games in Economics and Management Science. Cambridge University Press.

Elmaghraby, W. and P. Keskinocak (2003). Dynamic pricing in the presence of inventory considerations: Research overview, current practices, and future directions. Management Science 49(10), 1287-1309.

Fudenberg, D. and J. Tirole (1991). Game Theory. MIT Press, Cambridge, MA.

Gallego, G. and M. Hu (2014). Dynamic pricing of perishable assets under competition. Management Science 60(5), 1241-1259.

Gallego, G. and M. Talebian (2012). Demand learning and dynamic pricing for multi-version products. Journal of Revenue \& Pricing Management 11(3), 303-318. 
Gallego, G. and G. Van Ryzin (1997). A multiproduct dynamic pricing problem and its applications to network yield management. Operations Research 45(1), 24-41.

Gomes, D. and J. a. Saúde (2014). Mean field games models a brief survey. Dynamic Games and Applications 4(2), 110-154.

Gomes, D., R. M. Velho, and M.-T. Wolfram (2014). Socio-economic applications of finite state mean field games. Philosophical Transactions of the Royal Society of London A: Mathematical, Physical and Engineering Sciences 372(2028), 20130405.

Guéant, O., J.-M. Lasry, and P.-L. Lions (2011). Mean field games and applications. In Paris-Princeton Lectures on Mathematical Finance 2010, pp. 205-266. Springer.

Gutierrez, G. J. and X. He (2011). Life-cycle channel coordination issues in launching an innovative durable product. Production and Operations Management 20(2), 268-279.

Helmes, K., R. Schlosser, and M. Weber (2013). Optimal advertising and pricing in a class of general new-product adoption models. European Journal of Operational Research 229(2), $433-443$.

Huang, M., P. E. Caines, and R. P. Malhamé (2007). Large-population cost-coupled LQG problems with nonuniform agents: Individual-mass behavior and decentralized $\varepsilon$-Nash equilibria. Automatic Control, IEEE Transactions on 52(9), 1560-1571.

Huang, M., R. P. Malhamé, and P. E. Caines (2005). Nash equilibria for large-population linear stochastic systems of weakly coupled agents. In Analysis, Control and Optimization of Complex Dynamic Systems, pp. 215-252. Springer.

Huang, M., R. P. Malhamé, P. E. Caines, et al. (2006). Large population stochastic dynamic games: Closed-loop McKean-Vlasov systems and the Nash certainty equivalence principle. Communications in Information \&5 Systems 6(3), 221-252.

Iyer, K., R. Johari, and M. Sundararajan (2014). Mean field equilibria of dynamic auctions with learning. Management Science 60(12), 2949-2970.

Jiang, Z. and D. C. Jain (2012). A generalized Norton-Bass model for multigeneration diffusion. Management Science 58(10), 1887-1897.

Jing, B. (2011). Social learning and dynamic pricing of durable goods. Marketing Science $30(5), 851-865$.

Kalish, S. (1983). Monopolist pricing with dynamic demand and production cost. Marketing Science 2(2), 135-159. 
Kiesling, E., M. Günther, C. Stummer, and L. M. Wakolbinger (2012). Agent-based simulation of innovation diffusion: a review. Central European Journal of Operations Research 20(2), $183-230$.

Krishnan, T. V., F. M. Bass, and D. C. Jain (1999). Optimal pricing strategy for new products. Management Science 45(12), 1650-1663.

Lachapelle, A., J.-M. Lasry, C.-A. Lehalle, and P.-L. Lions (2013). Efficiency of the price formation process in presence of high frequency participants: A mean field game analysis. Mathematics and Financial Economics 10(3), 223-262.

Lachapelle, A., J. Salomon, and G. Turinici (2010). Computation of mean field equilibria in economics. Mathematical Models and Methods in Applied Sciences 20(04), 567-588.

Lachapelle, A. and M.-T. Wolfram (2011). On a mean field game approach modeling congestion and aversion in pedestrian crowds. Transportation Research Part B: Methodological 45(10), 1572-1589.

Laguzet, L. and G. Turinici (2015). Individual vaccination as Nash equilibrium in a SIR model with application to the 2009-2010 influenza A (H1N1) epidemic in France. Bulletin of Mathematical Biology 7r(10), 1955-1984.

Laguzet, L., G. Turinici, and G. Yahiaoui (2016). Equilibrium in an individual-societal SIR vaccination model in presence of discounting and finite vaccination capacity. In New Trends in Differential Equations, Control Theory and Optimization: Proceedings of the 8th Congress of Romanian Mathematicians, pp. 201-214.

Lasry, J.-M. and P.-L. Lions (2006a). Jeux à champ moyen. I: Le cas stationnaire. C. R., Math., Acad. Sci. Paris 343(9), 619-625.

Lasry, J.-M. and P.-L. Lions (2006b). Jeux à champ moyen. II: Horizon fini et contrôle optimal. C. R., Math., Acad. Sci. Paris 343(10), 679-684.

Lasry, J.-M. and P.-L. Lions (2007). Mean field games. Japanese Journal of Mathematics 2(1), 229-260.

Lasry, J.-M., P.-L. Lions, and O. Guéant (2008). Application of mean field games to growth theory.

Lucas, R. and B. Moll (2013). Knowledge growth and the allocation of time. Journal of Political Economy 122(1), 1-51.

Raman, K. and R. Chatterjee (1995). Optimal monopolist pricing under demand uncertainty in dynamic markets. Management Science 41(1), 144-162. 
Robinson, B. and C. Lakhani (1975). Dynamic price models for new-product planning. Management Science 21(10), 1113-1122.

Salvarani, F. and G. Turinici (2017). Optimal individual strategies for influenza vaccines with imperfect efficacy and durability of protection. Math. Biosci. Eng.. to appear.

Spann, M., M. Fischer, and G. J. Tellis (2014). Skimming or penetration? Strategic dynamic pricing for new products. Marketing Science 34(2), 235-249.

Sultan, F., J. U. Farley, and D. R. Lehmann (1990). A meta-analysis of applications of diffusion models. Journal of Marketing Research, 70-77.

Weintraub, G. Y., C. L. Benkard, and B. Van Roy (2008). Markov perfect industry dynamics with many firms. Econometrica 76(6), 1375-1411.

Weintraub, G. Y., C. L. Benkard, and B. Van Roy (2011). Industry dynamics: Foundations for models with an infinite number of firms. Journal of Economic Theory 146(5), 1965-1994.

Xie, J. and M. Sirbu (1995). Price competition and compatibility in the presence of positive demand externalities. Management Science 41(5), 909-926.

Yang, J. and Y. Xia (2013). A nonatomic-game approach to dynamic pricing under competition. Production and Operations Management 22(1), 88-103. 